TRANSACTIONS OF THE

AMERICAN MATHEMATICAL SOCIETY

Volume 184, Octobet 1973

\title{
ON THE UNIFORM CONVERGENCE OF QUASICONFORMAL MAPPINGS
}

BY

\author{
BRUCE PALKA( $\left.{ }^{1}\right)$
}

ABSTRACT. Let $D$ be a domain in extended Euclidean $n$-space with "smooth" boundary' and let $\left\{f_{j}\right\}$ be a sequence of $K$-quasiconformal mappings of $D$ into $R^{n}$ which converges uniformly on compact sets in $D$ to a quasiconformal mapping. This paper considers the que stion: When does the sequence $\left\{f_{j}\right\}$ converge uniformly on all of $D$ ? Geometric conditions on the domains $f_{j}(D)$ are given which are sufficient and, in many cases, necessary for uniform convergence. The particular case where $D$ is the unit ball in $R^{n}$ is examined to obtain analogues to classical convergence theorems for conformal mappings in the plane.

1. Introduction. In this paper, we continue the investigation, begun in [13], of the following question: given a domain $D$ in extended $n$-space with "smooth" boundary and given a sequence $\left\{f_{j}\right\}$ of $K$-quasiconformal mappings of $D$ which converges to a quasiconformal map uniformly on compact subsets of $D$, when can one infer that the convergence occurs uniformly on all of $D$ ? We give geometric conditions on the domains $D_{j}^{\prime}=f_{j}(D)$ which are sufficient and, in many cases, necessary for uniform convergence. We then consider the special case where $D$ is the unit ball in $R^{n}$ and where each $D_{j}^{\prime}$ is a Jordan domain to obtain analogues to classical the orems on conformal mappings in the plane. Many of the results in this paper generalize work of Gaier [3].

2. Notation and terminology. For $n \geq 2$ we denote by $\bar{R}^{n}$ the one-point compactification of $R^{n}$, Euclide an $n$-space: $\bar{R}^{n}=R^{n} \cup\{\infty\}$. The topology in $\bar{R}^{n}$ is given by a metric $q$, the chordal metric induced by stereographic projection. For a subset $A$ of $\bar{R}^{n}, \bar{A}, \partial A, C(A)$, and $q(A)$ will designate the closure, boundary, complement, and chordal diameter of $A$ respectively. For sets $A$ and $B$ in $\bar{R}^{n}$ we denote by $A \backslash B$ the difference set $A \cap C(B)$ and by $q(A, B)$ the chordal distance between $A$ and $B$. For $x \in R^{n}$ and $r>0, B^{n}(x, r)$ is the open (Euclidean) ball of radius $r$ with center at $x$ and we write $S^{n-1}(x, r)$ for $\partial B^{n}(x, r)$.

Received by the editors November 21, 1972.

AMS (MOS) subject classifications (1970). Primary 30A60; Secondary 30A74.

Key words and phrases. Quasiconformal mapping, uniform convergence, uniform domain, modulus of a path family, Fréchet distance.

(1) Portions of this paper are based upon the author's doctoral dissertation at the University of Michigan where he was partially supported by an NDEA Title IV Traineeship. 
We abbreviate: $B^{n}=B^{n}(0,1), S^{n-1}=S^{n-1}(0,1)$. The surface area of $S^{n-1}$ is denoted by $\omega_{n-1}$. A domain in $\bar{R}^{n}$ is a nonempty, open, connected subset of $\bar{R}^{n}$. A continuum is a closed connected set containing at least two points.

By a path in $\bar{R}^{n}$ we understand a continuous, nonconstant mapping of a closed line interval into $\bar{R}^{n}$. If $E, F$ and $G$ are subsets of $\bar{R}^{n}$, the notation $\Delta(E, F: G)$ is used for the family of all paths joining $E$ to $F$ in $G:$ a path $\gamma:[a, b] \rightarrow \bar{R}^{n}$ belongs to $\Delta(E, F: G)$ if and only if one endpoint belongs to $E$, one to $F$, and $\gamma(t)$ is in $G$ for each $t \in(a, b)$. For a family $\Gamma$ of paths in $\bar{R}^{n}, F(\Gamma)$ will denote the set of all nonnegative, extended-real-valued, Borel measurable functions $\rho$ on $\bar{R}^{n}$ such that $\int_{\gamma} \rho(x) d s \geq 1$ for each rectifiable $\gamma$ in $\Gamma$. The $n$-modulus of $\Gamma$, written $M(\Gamma)$, is defined by

$$
M(\Gamma)=\inf _{F(\Gamma)} \int_{R^{n}} \rho^{n}(x) d x .
$$

If $D$ and $D^{\prime}$ are domains in $\bar{R}^{n}$ and if $f$ is a homeomorphism of $D$ onto $D^{\prime}$, $f$ is said to be $K$-quasiconformal, $1 \leq K<\infty$, provided $M(\Gamma) / K \leq M(f(\Gamma)) \leq K M(\Gamma)$ for each path family $\Gamma$ in $D$. A homeomorphism is quasiconformal if it is $K$ quasiconformal for some $K$. A chordal isometry is a 1-quasiconformal map of $\bar{R}^{n}$ onto itself such that $q(f(x), f(y))=q(x, y)$ for all $x$ and $y$ in $\bar{R}^{n}$.

A domain $D$ in $\bar{R}^{n}$ is said to be quasiconformally collared if each $b$ in $\partial D$ has arbitrarily small neighborhoods $U$ such that $U \cap D$ can be mapped quasiconformally onto $B^{n}$. A quasiconformally collared domain has only finitely many boundary components, each of which is a compact $(n-1)$-dimensional manifold. Conversely, if a domain $D$ has only finitely many boundary components, each of which is an $(n-1)$-dimensional $C^{1}$-manifold, then $D$ is quasiconformally collared. For proofs of the above statements, the reader is referred to [9], where further discussion of quasiconformal collaredness is to be found. We note, in particular, that $B^{n}$ is quasiconformally collared.

If $D$ and $D^{\prime}$ are domains in $\bar{R}^{n}$ and if $f$ is a homeomorphism of $D$ onto $D^{\prime}$, we denote by $C(f ; b)$ the cluster set of $f$ at the point $b$ in $\partial D$. Thus $C(f ; b) C$ $\partial D^{\prime}$ and $b^{\prime}$ belongs to $C(f ; b)$ if and only if there is a sequence $\left\{b_{j}\right\}$ in $D$ such that $b_{j} \rightarrow b$ and $f\left(b_{j}\right) \rightarrow b^{\prime}$.

3. The function $\delta(r ; D)$. Let $D$ be a domain in $\bar{R}^{n}$. For each $r \in(0,1]$ we denote by $K_{r}(D)$ the collection of all connected subsets $F$ of $D$ with $q(F) \geq r$. We define

$$
\delta(r ; D)=\inf _{F_{1}, F_{2} \in K_{r}(D)} M\left(\Delta\left(F_{1}, F_{2}: D\right)\right)
$$

if $K_{r}(D) \neq \varnothing$ and $\delta(r ; D)=\infty$ otherwise. The function $\delta(r ; D)$ is clearly non- 
decreasing on $(0,1]$. If $\delta(r ; D) \equiv 0$ on $(0,1]$ we set $\delta(D)=1$; otherwise we let

$$
\delta(D)=\inf \{r \in(0,1] \mid \delta(r ; D)>0\} \text {. }
$$

For example, recent unpublished results of Gehring [6] can be used to show that $\delta\left(r ; B^{n}\right) \geq C \log \left(4 r^{2}+1\right)$ for all $r \in(0,1]$, where $C$ is a positive constant depending only on $n$. Thus $\delta\left(B^{n}\right)=0$.

We intend to use the function $\delta(r ; D)$ as an index to measure the regularity of the boundary of $D$. A domain $D$ is called a uniform domain if and only if $\delta(D)=0$. Uniform domains have been studied in some detail in [10] and [12], where it has been show $n$ that the boundary of a uniform domain is reasonably regular. More precisely, if $D$ is a uniform domain and if $b \in \partial D$, then $b$ has arbitrarily small neighborhoods $U$ such that $U \cap D$ has only finitely many components. Conversely, domains with smooth boundaries are uniform domains-for instance, quasiconformally collared domains are uniform domains. Furthermore, the function $\delta(r ; D)$ determines whether a quasiconformal mapping of a quasiconformally collared domain onto $D$ has reasonable boundary behavior. We have

Theorem 1. Let $D_{0}$ be a quasiconformally collared domain in $\bar{R}^{n}$ and let $f$ be a K-quasiconformal map of $D_{0}$ onto a domain $D$. Then

$$
\delta(D)=\sup _{b \in \partial D_{0}} q(C(f ; b))
$$

Proof. Set $L=\sup \left\{q(C(f ; b)) \mid b \in \partial D_{0}\right\}$.

We show first that $\delta(D) \leq L$. For this let $\epsilon>0$ and choose $t>0$ such that for all $x$ and $y$ in $D_{0}$ with $q(x, y)<t$ we have

$$
q(f(x), f(y))<L+\epsilon / 2 \text {. }
$$

Since $D_{0}$ is a uniform domain there is a $\delta_{0}>0$ such that

$$
M\left(\Delta\left(E, F: D_{0}\right)\right) \geq \delta_{0}
$$

whenever $E$ and $F$ belong to $K_{t}\left(D_{0}\right)$. If $K_{L+\epsilon}(D)=\varnothing$, then trivially $\delta(D) \leq$ $L+\epsilon$. Otherwise let $E^{\prime}, F^{\prime}$, be sets in $K_{L+\epsilon}(D)$. By $(4), E=f^{-1}\left(E^{\prime}\right)$ and $F=f^{-1}\left(F^{\prime}\right)$ belong to $K_{t}\left(D_{0}\right)$. It follows that

$$
M\left(\Delta\left(E^{\prime}, F^{\prime}: D\right)\right) \geq(1 / K) M\left(\Delta\left(E, F: D_{0}\right)\right) \geq \delta_{0} / K
$$

and, there fore, that

$$
\delta(L+\epsilon ; D) \geq \delta_{0} / K>0 .
$$

We infer that $\delta(D) \leq L+\epsilon$ and, letting $\epsilon \rightarrow 0$, that $\delta(D) \leq L$.

To obtain the reverse inequality we fix $b \in \partial D_{0}$ and set $r=q(C(f ; b))$. If 
$r>\delta(D)$ we can find a continuum $A$ in $D$ with $r^{\prime}=q(A)>\delta(D)$. We set $r^{\prime \prime}=$ $\min \left\{r, r^{\prime}\right\}$. By definition,

$$
\delta\left(r^{\prime \prime} ; D\right)=d>0 .
$$

Next we can pick a neighborhood $U$ of $b$ such that $C=U \cap D_{0}$ is connected and, moreover, such that

$$
M\left(\Delta\left(C, f^{-1}(A): D_{0}\right)\right)<d / K
$$

But then $M(\Delta(f(C), A: D))<d$. Since $q(A) \geq r^{\prime \prime}$ we infer from $(6)$ that $q(f(C))<r^{\prime \prime}$. On the other hand, $C(f ; b) \subset \overline{f(C)}$, whence

$$
q(C(f ; b)) \leq q\left(\overline{f(C))}=q(f(C))<r^{\prime \prime} \leq r,\right.
$$

a contradiction. We conclude that $r=q(C(f ; b)) \leq \delta(D)$ and, taking the supremum over $b$ in $\partial D_{0}$, that $L \leq \delta(D)$. This completes the proof of Theorem 1 .

As an immediate corollary to Theorem 1 we obtain the following result of Näkki [9].

Corollary 1. Let $D_{0}$ be a quasiconformally collared domain in $\bar{R}^{n}$ and let $f$ map $D_{0}$ quasiconformally onto a domain $D$. $f$ has an extension to a continuous map of $\bar{D}_{0}$ onto $\bar{D}$ if and only if $D$ is a uniform domain.

4. Convergence theorems for collared domains. In this section we make the following basic assumptions: $D$ is a fixed quasiconformally collared domain in $\bar{R}^{n}$; for $j=1,2, \ldots, f_{j}$ is a $K$-quasiconformal mapping of $D$ onto a domain $D_{j}^{\prime} ;\left\{f_{j}\right\}_{j=1}^{\infty}$ converges uniformly on compact subsets of $D$ to a $K$-quasiconformal map $f^{j}$. We ask for conditions under which we can conclude that $f_{j} \rightarrow f$ uniformly on all of $D$. Simple examples lead one to expect that, in many cases, the uniform convergence of $\left\{f_{j}\right\}_{j=1}^{\infty}$ will depend upon the regularity of the boundaries of the domains $D_{j}^{\prime}$. We intend to use the functions $\delta\left(r ; D_{j}^{\prime}\right)$ to give this statement precise meaning. We begin with a lemma.

Lemma 1. Under the basic assumptions above suppose that, for each $r \in(0,1]$,

$$
\limsup _{j \rightarrow \infty} \delta\left(r ; D_{j}^{\prime}\right)>0
$$

Then $f$ bas an extension to a continuous map on $\bar{D}$.

Proof. Fix $b \in \partial D$. It suffices to show that $f$ has a limit as $x \rightarrow b, x \in D$. Let $\epsilon>0$ be given. We need only show that $b$ has a neighborhood $U$ such that $q(f(x), f(y))<\epsilon$ for $x, y$ in $U \cap D$. For this fix a continuum $A$ in $D$ and set

$$
r=\min \left\{\frac{\epsilon}{3}, \inf _{j \geq 1} q\left(f_{j}(A)\right)\right\}>0 \text {. }
$$


Because of (8) there is a $d>0$ such that

$$
\delta\left(r ; D_{j}^{\prime}\right)>d
$$

for infinitely many $j$. Next choose a neighborhood $U$ of $b$ such that $C=U \cap D$ is connected and such that

$$
M(\Delta(A, C: D))<d / K .
$$

Now fix $x, y$ in $C$. We can choose an index $j$ for which (10) is valid and, furthermore, for which

$$
q\left(f_{j}(x), f(x)\right)<\epsilon / 3, \quad q\left(f_{j}(y), f(y)\right)<\epsilon / 3 .
$$

From (11) we infer that $M\left(\Delta\left(f_{j}(A), f_{j}(C): D_{j}^{\prime}\right)\right)<d_{\text {. Since }} q\left(f_{j}(A)\right) \geq r,(10)$ implies that

$$
q\left(f_{j}(x), f_{j}(y)\right) \leq q\left(f_{j}(C)\right)<r \leq \epsilon / 3 .
$$

Combining (12) and (13) we obtain $q(f(x), f(y))<\epsilon$. Since $x$ and $y$ were arbitrary points of $C=U \cap D$ the result follows.

Lemma 1 provides the key to determining a sufficient condition for the uniform convergence of $\left\{f_{j}\right\}_{j=1}^{\infty}$.

Theorem 2. Under the basic assumptions above suppose that, for each $r \in(0,1]$,

$$
\underset{j \rightarrow \infty}{\liminf _{j \rightarrow \infty} \delta\left(r ; D_{j}^{\prime}\right)>0}
$$

Then $f_{j} \rightarrow f$ uniformly on $D$.

Proof. If the result is false we may assume-after passing to a subsequence and relabeling-that there is a sequence $\left\{b_{j}\right\}_{j=1}^{\infty}$ in $D$ such that $q\left(f_{j}\left(b_{j}\right), f\left(b_{j}\right)\right) \geq$ $\epsilon>0$ for all $j \geq 1$. We may assume further that $b_{j} \rightarrow b$, where $b$ necessarily belongs to $\partial D$.

Fix a continuum $A$ in $D$ and set

$$
r=\min \left\{\frac{\epsilon}{3}, \inf _{j} q\left(f_{j}(A)\right)\right\}>0 .
$$

By (14) there is a $d>0$ and an index $j_{0}$ such that

$$
\delta\left(r ; D_{j}^{\prime}\right)>d
$$

for $j \geq j_{0}$. Next pick a neighborhood $U$ of $b$ such that $C=U \cap D$ is connected, such that

$$
M(\Delta(A, C: D))<d / K
$$

and, finally, such that 


$$
q(f(C))<\epsilon / 3
$$

(18) is possible since, by Lemma $1, f$ extends to a continuous map on $\bar{D}$.

Now fix a point $b_{0}$ in $C$ and fix $j \geq j_{0}$ such that $b_{j}$ belongs to $C$ and such that

$$
q\left(f_{j}\left(b_{0}\right), f\left(b_{0}\right)\right)<\epsilon / 3 .
$$

In view of (17), $M\left(\Delta\left(f_{j}(A), f_{j}(C): D_{j}^{\prime}\right)\right)<d$ and, since $q\left(f_{j}(A)\right) \geq r$, it follows from (16) that

$$
q\left(f_{j}\left(b_{0}\right), f_{j}\left(b_{j}\right)\right) \leq q\left(f_{j}(C)\right)<r \leq \epsilon / 3 \text {. }
$$

On the other hand, we infer from (18) and (19) that

$$
\begin{aligned}
q\left(f_{j}\left(b_{0}\right), f_{j}\left(b_{j}\right)\right) & \geq q\left(f_{j}\left(b_{j}\right), f\left(b_{j}\right)\right)-q\left(f\left(b_{j}\right), f\left(b_{0}\right)\right)-q\left(f\left(b_{0}\right), f_{j}\left(b_{0}\right)\right) \\
& \geq \epsilon-\epsilon / 3-\epsilon / 3=\epsilon / 3,
\end{aligned}
$$

contradicting (20). We conclude that $f_{j} \rightarrow f$ uniformly on $D$. Theorem 2 has thus been established.

Condition (14) by itself is certainly not a necessary condition for the uniform convergence of $\left\{f_{j}\right\}_{j=1}^{\infty}$. For example, if $f$ is a quasiconformal map of $B^{n}$ onto a nonuniform domain $D^{\prime}$ and if we set $f_{j}=f$ for $j=1,2, \ldots$ then trivially $f_{j} \rightarrow$ $f$ uniformly on $B^{n}$, while (14) fails to hold. Clearly the best we can expect is that (14) is necessary in the case that $f$ has a continuous extension to $\bar{D}$.

Theorem 3. Under the basic assumptions above suppose that $f_{j} \rightarrow f$ uniformly on $D$ and that $f$ extends to a continuous map on $\bar{D}$. Then $\lim \inf _{j \rightarrow \infty} \delta\left(r ; D_{j}^{\prime}\right)>0$ for each $r \in(0,1]$.

Proof. Fix $r \in(0,1]$. Because $f$ is uniformly continuous on $D$ we can find $t>0$ such that

$$
q(f(x), f(y))<r / 3
$$

whenever $x$ and $y$ in $D$ satisfy $q(x, y)<t$. Since $D$ is a uniform domain there is a $d>0$ such that

$$
M(\Delta(E, F: D)) \geq d
$$

whenever $E$ and $F$ belong to $K_{t}(D)$.

Because $f_{i j} \rightarrow f$ uniformly on $D$ we can fix an index $j_{0}$ so that

$$
q\left(f_{j}(x), f(x)\right)<r / 6
$$

for all $x$ in $D$, whenever $j \geq j_{0}$. Together (21) and (23) imply that

$$
q\left(f_{j}(x), f_{j}(y)\right)<5 r / 6,
$$


whenever $j \geq j_{0}$ and $x$ and $y$ are points of $D$ satisfying $q(x, y)<t$.

Now fix $j \geq j_{0}$ and let $E^{\prime}, F^{\prime}$ be in $K_{r}\left(D_{j}^{\prime}\right)$. Then, by (24), $E=f_{j}^{-1}\left(E^{\prime}\right)$ and $F=f_{j}^{-1}\left(F^{\prime}\right)$ belong to $K_{i}(D)$ and we infer from (22):

$$
M\left(\Delta\left(E^{\prime}, F^{\prime}: D_{j}^{\prime}\right)\right) \geq(1 / K) M(\Delta(E, F: D)) \geq d / K .
$$

It follows that $\delta\left(r ; D_{j}^{\prime}\right) \geq d / K$ and, since this holds for each $j \geq j_{0}$, that lim inf ${ }_{j \rightarrow \infty} \delta\left(r ; D_{j}^{\prime}\right) \geq d / K>0$. This completes the proof of Theorem 3 .

As an immediate corollary to Corollary 1 and Theorems 2 and 3, we obtain the following result (cf. [11]):

Corollary 2. Let $D$ be a quasiconformally collared domain in $\bar{R}^{n}$. For $j=$ $1,2, \ldots$ let $f_{j}$ be a continuous map of $\bar{D}$ into $\bar{R}^{n}$ such that $f_{j}$ maps $D K$. quasiconformally onto a domain $D_{j}^{\prime}$. Assume that $\left\{f_{j}\right\}_{j=1}^{\infty}$ converges uniformly on compact subsets of $D$ to a $K$-quasiconformal map. Then $\left\{f_{j}\right\}_{j=1}^{\infty}$ converges uniformly on $\bar{D}$ if and only if, for each $r \in(0,1]$ inf $_{j \geq 1} \delta\left(r ; D_{j}^{\prime}\right)>0$.

As the simplest example illustrating the content of Theorem 2, consider the case $n=2, K=1, D=B^{2}$. Let

$$
\begin{aligned}
& D^{\prime}=\{z=x+i y|| x|<1,| y \mid<1\}, \\
& D_{j}^{\prime}=D^{\prime} \backslash\{z=x+i y \mid x=1-1 / j, 0 \leq y \leq 1\}, \\
& D_{j}^{\prime \prime}=D^{\prime} \backslash\{z=x+i y \mid x=1-1 / j, 1-1 / j \leq y \leq 1\}
\end{aligned}
$$

for $j=2,3, \ldots$.
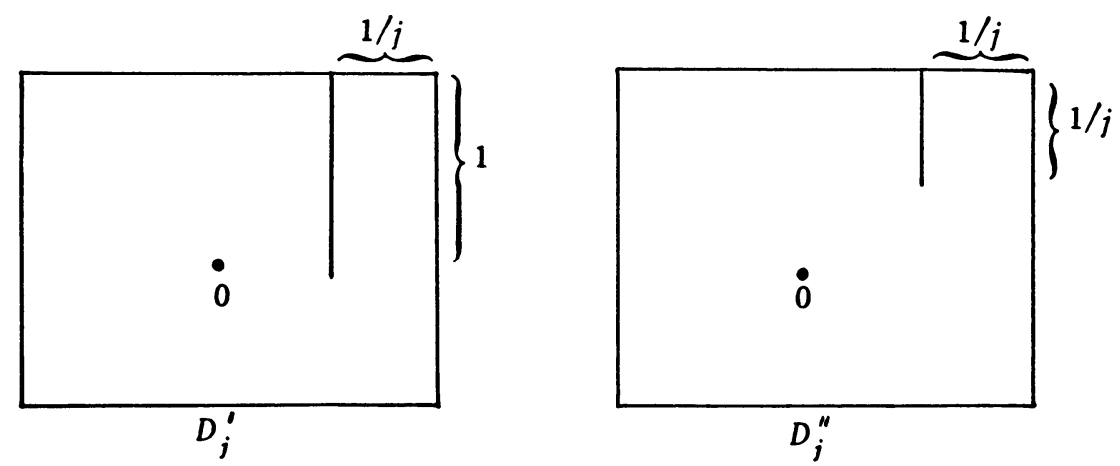

Let $f_{j}$ be the conformal map of $B^{2}$ onto $D_{j}^{\prime}$ with $f_{j}(0)=0, f_{j}^{\prime}(0)>0$. Let $g_{j}$ be the corresponding map of $B^{2}$ onto $D_{j}^{\prime \prime}$. Each of the sequences $\left\{f_{j}\right\},\left\{g_{j}\right\}$ converges to the map $f$ of $B^{2}$ onto $D^{\prime}$ with $f(0)=0, f^{\prime}(0)>0$, uniformly on compact subsets of $B^{2}$. In the first case condition (14) fails and convergence is not uniform on $B^{2}$, while in the second case (14) holds and convergence is uniform. 
5. Inverse mappings. In this section we prove an $n$-dimensional analogue to theorems of Farrell [2] and Gaier [3].

Theorem 4. Let $D$ be a quasiconformally collared domain in $\bar{R}^{n}$. For $j=$ $1,2, \ldots$, let $f_{j}$ be a K-quasiconformal map of $D$ onto a domain $D_{j}^{\prime}$ such that $f_{j} \rightarrow f$ uniformly on compact subsets of $D$, where $f$ maps $D K$-quasiconformally onto a domain $D^{\prime}$. Write $b_{j}=f_{j}^{-1}, b=f^{-1}$. Assume that $D^{\prime} \subset D_{j}^{\prime}$ for all $j \geq 1$ and that $f$ bas an extension to a continuous map of $\bar{D}$ onto $\bar{D}^{\prime}$. 'Then $b_{j} \rightarrow b$ uniformly on $D^{\prime}$. In particular, the conclusion follows if $D^{\prime} \subset D_{j}^{\prime}$ for all $j \geq 1$ and if condition (8) is satisfied.

Proof. Theorem 21.10 of [15] implies that $b_{j} \rightarrow b$ uniformly on compact subsets of $D^{\prime}$. If the convergence is not uniform on all of $D^{\prime}$ then-after passing to a subsequence and relabeling-we may assume that there is a sequence $\left\{b_{j}\right\}_{j=1}^{\infty}$ in $D^{\prime}$ such that

$$
q\left(b_{j}\left(b_{j}\right), b\left(b_{j}\right)\right) \geq \epsilon>0
$$

for all $j \geq 1$. We may assume that $b_{j} \rightarrow b^{\prime}$ and that $b\left(b_{j}\right) \rightarrow b$, where necessarily $b \in \partial D$ and $b^{\prime} \in \partial D^{\prime}$. Fix a continuum $A$ in $D^{\prime}$ and set

$$
r=\min \left\{\frac{\epsilon}{3}, \underset{j}{\inf } q\left(b_{j}(A)\right)\right\}>0 .
$$

Since $D$ is a uniform domain

$$
\delta(r ; D)=d>0
$$

and we can pick a neighborhood $U^{\prime}$ of $b^{\prime}$ such that

$$
M\left(\Delta\left(A, U^{\prime}: \bar{R}^{n}\right)\right)<d / K .
$$

By hypothesis $f$ is continuous at $b$ and $f(b)=\lim _{j \rightarrow \infty} f\left(b\left(b_{j}\right)\right)=\lim _{j \rightarrow \infty} b_{j}=b^{\prime}$. We can, therefore, choose a neighborhood $U$ of $b$ such that

$$
q(U)<\epsilon / 3 \text {, }
$$

$C=U \cap D$ is connected and $C^{\prime}=f(C) \subset U^{\prime}$. We may assume that $b\left(b_{j}\right) \in C$ for all $j \geq 1$ and, hence, that $b_{j} \in C^{\prime}$ for all $j \geq 1$.

Now fix a point $x_{0} \in C^{\prime}$ and an index $j$ for which

$$
q\left(b_{j}\left(x_{0}\right), b\left(x_{0}\right)\right)<\epsilon / 3 .
$$

Then $M\left(\Delta\left(A, C^{\prime}: D_{j}^{\prime}\right)\right) \leq M\left(\Delta\left(A, U^{\prime}: \bar{R}^{n}\right)\right)<d / K$ from which we infer $M\left(\Delta\left(b_{j}(A), b_{j}\left(C^{\prime}\right): D\right)\right)<d$. Since $q\left(b_{j}(A)\right) \geq r$ it follows from (27) that

$$
q\left(b_{j}\left(x_{0}\right), b_{j}\left(b_{j}\right)\right) \leq q\left(b_{j}\left(C^{\prime}\right)\right)<r \leq \epsilon / 3 .
$$


On the other hand (25), (29), and (30) give

$$
\begin{aligned}
q\left(b_{j}\left(x_{0}\right), b_{j}\left(b_{j}\right)\right) & \geq q\left(b_{j}\left(b_{j}\right), b\left(b_{j}\right)\right)-q\left(b\left(b_{j}\right), b\left(x_{0}\right)\right)-q\left(b\left(x_{0}\right), b_{j}\left(x_{0}\right)\right) \\
& \geq \epsilon-\epsilon / 3-\epsilon / 3=\epsilon / 3,
\end{aligned}
$$

contradicting (31). Thus $b_{j} \rightarrow b$ uniformly on $D^{\prime}$ and the proof of Theorem 4 is complete.

We conjecture that Theorem 4 remains true if the assumption that $f$ has a continuous extension to $\bar{D}$ is deleted. For $n=2, K=1, D=B^{2}$ this is the result of Gaier mentioned above [3, Theorem 14]. In fact his proof establishes the result for $K>1$ as well. However, it utilizes techniques from the theory of prime ends which have no obvious counterparts in higher dimensions. We have been able to establish the stronger result only for the special case where $D^{\prime}=$ $D_{j}^{\prime}$ for all $j$. (See [11].)

6. The function $a(r ; S)$. In the remainder of this paper we restrict our attention to the case where $D=B^{n}$ and where each $D_{j}^{\prime}, j \geq 1$, is a Jordan domain, that is, $\partial D_{j}^{\prime}$ is homeomorphic to $S^{n-1}$. We intend to reformulate Corollary 2 in this special case in order, first of all, to give a metric characterization of the condition (14) and, secondly, to give $n$-dimensional versions of two classical theorems about conformal mappings. We recall that a quasiconformal map $f$ of $B^{n}$ onto a Jordan domain $D^{\prime}$ has an extension to a homeomorphism of $\bar{B}^{n}$ onto $\bar{D}^{\prime}$. (See Theorem 17.20 of [15].)

Let $S$ be a homeomorph of $S^{n-1}$ in $\bar{R}^{n}$. If $\Sigma \subset S$ is any homeomorph of $S^{n-2}$ -for $n=2, \Sigma$ will consist of two points-the Jordan-Brower separation theorem states that $S \backslash \Sigma$ consists of two components, $C_{1}(\Sigma)$ and $C_{2}(\Sigma)$. For each such $\Sigma \subset S$ we set

$$
a(\Sigma)=\min \left\{q\left(C_{1}(\Sigma)\right), q\left(C_{2}(\Sigma)\right)\right\}
$$

and, for $r \in(0,1]$, we define

$$
\alpha(r ; S)=\sup _{q(\Sigma) \leq r} a(\Sigma) .
$$

The function $a(r ; S)$ is nondecreasing on $(0,1]$. Moreover, if $b$ is a chordal isometry it is obvious that

$$
\alpha(r ; S)=\alpha(r ; b(S))
$$

for $r \in(0,1]$.

Roughly speaking $a(r, S)$ measures the "bulges" in $S$. For example, if $S$ is a quasiconformal sphere in $\bar{R}^{n}$, that is, if $S$ is the image of $S^{n-1}$ under a quasiconformal mapping of $\bar{R}^{n}$ onto itself, then there is a constant $C \in[1, \infty)$ such that 


$$
a(r ; S) \leq C r
$$

for all $r \in(0,1]$. For a proof the reader is referred to [12]. In fact, when $n=2$, condition (33) is also a sufficient condition for $S$ to be a quasiconformai circle. (See [8].) In general we can make the following observation about $a(r ; S)$ :

Lemma 2. Let $S$ be a bomeomorph of $S^{n-1}$ in $\bar{R}^{n}$. Then

$$
\lim _{r \rightarrow 0^{+}} a(r ; S)=0 \text {. }
$$

Proof. Fix a homeomorphism $f$ of $S^{n-1}$ onto $S$. Let $\epsilon>0$ be given. Choose $t \in(0,1)$ so that

$$
q(f(x), f(y))<\epsilon / 2
$$

for $x$ and $y$ in $S^{n-1}$ with $|x-y| \leq t$. Next, choose $r_{0} \in(0,1)$ such that

$$
\left|f^{-1}\left(x^{\prime}\right)-f^{-1}\left(y^{\prime}\right)\right|<t
$$

if $x^{\prime}$ and $y^{\prime}$ in $S$ satisfy $q\left(x^{\prime}, y^{\prime}\right) \leq r_{0}$. Now fix $r \in\left(0, r_{0}\right]$ and let $\Sigma$ be a homeomorph of $S^{n-2}$ in $S$ with $q(\Sigma) \leq r$. Choose $b^{\prime} \in \Sigma$ and write $b=f^{-1}\left(b^{\prime}\right)$. From (36) we can infer $f^{-1}(\Sigma) \subset \bar{B}^{n}(b, t)$. Since $S^{n-1} \backslash \bar{B}^{n}(b, t)$ cannot meet both $f^{-1}\left(C_{1}(\Sigma)\right)$ and $f^{-1}\left(C_{2}(\Sigma)\right)$ we may assume that $f^{-1}\left(C_{1}(\Sigma)\right)$ is contained in $\bar{B}^{n}(b, t) \cap S^{n-1}$. By $(35), a(\Sigma) \leq q\left(C_{1}(\Sigma)\right)<\epsilon$. It follows that $a(r ; S) \leq \epsilon$ and this for all $r \in\left(0, r_{0}\right]$. The conclusion now follows.

7. Preliminary lemmas. Before we can proceed with the discussion of uniform convergence we require several technical lemmas. The first is a simple consequence of Remark 7.5 in [15] and we omit its proof.

Lemma 3. Let $0<s<r \leq 1$ and let $A$ and $B$ be nonempty sets in $\bar{R}^{n}$ with $q(A) \leq s$ and $q(A, B) \geq r$. Then

$$
M\left(\Delta\left(A, B: \bar{R}^{n}\right)\right) \leq \omega_{n-1}(\log (r / s))^{1-n} .
$$

Lemma 4. Let $f$ be a continuous map of $\bar{B}^{n}$ into $\bar{R}^{n}$ which maps $B^{n} K$. quasiconformally onto a domain $D$. Let $E$ be a continuum in $\bar{B}^{n}$. Then $f(E)$ is a continuum.

Proof. We must show that $f(E)$ does not reduce to a point. This is obviously true if $E \cap B^{n} \neq \varnothing$, so we may assume that $E \subset S^{n-1}$. Choose a continuum $E^{\prime}$ in $B^{n}$ and set $M=M\left(\Delta\left(E, E^{\prime}: B^{n}\right)\right)$. By a recent result of Gehring [6], $M \geq$ $1 / 2 M\left(\Delta\left(E, E^{\prime}: \bar{R}^{n}\right)\right)>0$, since $\bar{R}^{n}$ is a uniform domain. If $f(E)$ reduces to a single point we can select an open set $U$ such that $f(E) \subset U$, while $M\left(\Delta\left(f\left(E^{\prime}\right), U: \bar{R}^{n}\right)\right)<M / K$. Write $\Gamma^{\prime}=\Delta\left(f\left(E^{\prime}\right), U \cap D: D\right), \Gamma=f^{-1}\left(\Gamma^{\prime}\right)$. Then $M(\Gamma) \leq K M\left(\Gamma^{\prime}\right)<M$. On the other hand, each path in $\Delta\left(E, E^{\prime}: B^{n}\right)$ contains a 
subpath in $\Gamma$ and it follows from Theorem 6.4 of [15] that $M \leq M(\Gamma)<M$, a contradiction. We conclude that $f(E)$ cannot reduce to a point and Lemma 4 is established.

For $n \geq 2$ and for $K \in[1, \infty)$ let $\theta_{n, K}$ be the function defined on $(0, \infty)$ by

$$
\theta_{n, K}(t)=\exp \left(\left[K \omega_{n-1} / t\right]^{1 /(n-1)}\right) .
$$

$\theta_{n, K}$ is a decreasing function and, clearly, $\theta_{n, K}(t) \geq 1$.

Lemma 5. Let $f$ be a continuous map of $\bar{B}^{n}$ into $\bar{R}^{n}$ which maps $B^{n} K$ quasiconformally onto a domain $D$. Let $E$ and $F$ be disjoint continua in $\bar{B}^{n}$ and set $M=M\left(\Delta\left(E, F: B^{n}\right)\right)$. Then

$$
q(f(E), f(F)) \leq q(f(E)) \theta_{n, K}(M) .
$$

Proof. Since $E$ and $F$ are disjoint closed sets $M<\propto$. Moreover, again using an unpublished result of Gehring [6],

$$
M \geq 1 / 2 M\left(\Delta\left(E, F: \bar{R}^{n}\right)\right)>0 .
$$

Set $r=q(f(E), f(F))$ and $s=q(f(E))$. If $r \leq s$ there is nothing to prove. In light of Lemma 4 we may assume that $0<s<r \leq 1$. Now fix $\in \in(0,(r-s) / 2)$ and let $U$ and $V$ be open sets with $f(E) \subset U$ and $f(F) \subset V$ satisfying

$$
q(U)<s+\epsilon, \quad q(U, V)>r-\epsilon .
$$

Write $\Gamma^{\prime}=\Delta(U \cap D, V \cap D: D)$ and $\Gamma=f^{-1}\left(\Gamma^{\prime}\right)$. By Lemma 3

$$
M\left(\Gamma^{\prime}\right) \leq \omega_{n-1}(\log (r-\epsilon) /(s+\epsilon))^{1-n} .
$$

On the other hand, each path in $\Delta\left(E, F: B^{n}\right)$ contains a subpath belonging to $\Gamma$. By Theorem 6.4 of $[15], M(\Gamma) \geq M$, whence

$$
M\left(\Gamma^{\prime}\right) \geq M(\Gamma) / K \geq M / K
$$

Combining (39) and (40) and letting $\epsilon \rightarrow 0$, we obtain $M \leq K \omega_{n-1}(\log (r / s))^{1-n}$, which, in tum, yields (37). This completes the proof of Lemma 5.

Finally, we require the following result:

Lemma 6. Let $f$ be a K-quasiconformal map of $B^{n}$ into $\bar{R}^{n}$. Fix $b \in S^{n-1}$. For $t \in(0,1)$ set

$$
U_{t}=f\left(B^{n} \cap B^{n}(b, t)\right), \quad S_{t}=f\left(B^{n} \cap S^{n-1}(b, t)\right) .
$$

If $\infty \notin U_{t}$ then there is a $\rho \in\left(t^{2}, t\right)$ such that $q\left(S_{\rho}\right) \leq C_{1}\left[m\left(U_{t}\right) / \log (1 / t)\right]^{1 / n}$, where $m$ denotes Lebesgue measure and $C_{1}$ is a constant depending only on $n$ and $K$.

Proof. For $r \in\left(t^{2}, t\right)$ let $\sigma(r)=q\left(S_{r}\right)$. By a result of Gehring (cf. Lemma 8 
of [5]): $\int_{t^{2}}^{t} \sigma^{n}(r) d r / r \leq C_{0} m\left(U_{t}\right)$, where $C_{0}$ is a constant depending only on $n$ and $K$. Thus, for some $\rho \in\left(t^{2}, t\right)$,

$$
\sigma(\rho)=q\left(S_{\rho}\right) \leq C_{1}\left[m\left(U_{t}\right) / \log (1 / t)\right]^{1 / n}
$$

where $C_{1}=\left(2 C_{0}\right)^{1 / n}$.

8. The case of Jordan domains. We are now prepared to proceed with the reformulation of Corollary 2 in the case where each $f_{j}, j \geq 1$, is a homeomorphism of $\bar{B}^{n}$ onto $\bar{D}_{j}^{\prime}$. We begin with a lemma.

Lemma 7. For $j=1,2, \cdots$, let $f_{j}$ be a bomeomorpbism of $\bar{B}^{n}$ into $\bar{R}^{n}$ mapping $B^{n} K$-quasiconformally onto a domain $D_{j}^{\prime}$. Assume that $\left\{f_{j}\right\}_{j=1}^{\infty}$ converges uniformly on compact subsets of $B^{n}$ to a K-quasiconformal mapping $f$. Assume, furtber, that

$$
\lim _{r \rightarrow 0^{+}} \sup _{j \geq 1} \alpha\left(r ; \partial D_{j}^{\prime}\right)=0
$$

Then $\left\{f_{j}\right\}_{j=1}^{\infty}$ converges uniformly on $\bar{B}^{n}$.

Proof. Before commencing with the proof we make some preliminary remarks. Using (32) we may assume that $f(0)=\infty$. Let $F=\bar{B}^{n}(0,1 / 2), F_{1}=\bar{B}^{n}(0,1 / 4)$, $R=B^{n} \backslash F$, and $R_{1}=B^{n} \backslash F_{1}$. For all sufficiently large $j$ we have

$$
f_{j}\left(F_{1}\right) \subset f(F)
$$

and

$$
f\left(F_{1}\right) \subset f_{j}(F)
$$

From (42) we infer that there is a $d>0$ such that

$$
q\left(f_{j}\left(F_{1}\right), \partial D_{j}^{\prime}\right) \geq d
$$

for all $j \geq 1$. We may clearly assume that (43) is likewise valid for all $j \geq 1$. Then

$$
m\left(f_{j}(R)\right) \leq m\left(f\left(R_{1}\right)\right)=A<\infty
$$

for all $j \geq 1$, where $m$ denotes Lebesgue measure.

We now proceed with the proof of Lemma 7. By Theorem 20.3 of [15] we need only show that $\mathcal{F}=\left\{f_{j}\right\}_{j=1}^{\infty}$ is equicontinuous on $\bar{B}^{n}$. Moreover, by Theorem 1 of [13] this will be the case provided $\left.\mathcal{F}\right|_{s^{n-1}}$ is equicontinuous on $s^{n-1}$. Thus it suffices to demonstrate that $\left.\mathfrak{F}\right|_{s^{n-1}}$ is equicontinuous at each fixed $b \in S^{n-1}$.

Fix $b \in S^{n-1}$ and $\epsilon>0$. Choose a continuum $E$ in $\left.S^{n-1} \backslash b\right\}$. Then, for $j \geq 1, q\left(f_{j}\left(F_{1}\right), f_{j}(E)\right) \geq q\left(f_{j}\left(F_{1}\right), \partial D_{j}^{\prime}\right) \geq d$ in view of (44). Lemma 5 implies that 


$$
q\left(f_{j}(E)\right) \geq \theta_{n, K}(M) / d=d^{\prime}>0
$$

for each $j \geq 1$, where $M=M\left(\Delta\left(E, F_{1}: B^{n}\right)\right)$.

Using (41) we can fix $r \in(0,1)$ such that

$$
\alpha\left(r ; \partial D_{j}^{\prime}\right)<\min \left\{\epsilon, d^{\prime}\right\}
$$

for $j \geq 1$. Next choose $t \in(0,1 / 4)$ so that $E \cap \bar{B}^{n}(b, t)=\varnothing$ and so that

$$
C_{1}[A / \log (1 / t)]^{1 / n}<r,
$$

where $C_{1}$ is the constant in Lemma 6 .

Finally, fix $x \in S^{n-1} \cap B^{n}\left(b, t^{2}\right)$ and $j \geq 1$. Lemma 6 asserts the existence of $\rho \in\left(t^{2}, t\right)$ such that with $S_{\rho}=f_{j}\left(S^{n-1}(b, \rho) \cap B^{n}\right)$ :

$$
q\left(S_{\rho}\right) \leq C_{1}\left[m\left(f_{j}(R)\right) / \log (1 / t)\right]^{1 / n} \leq C_{1}[A / \log (1 / t)]^{1 / n}<r .
$$

Then $\Sigma=f_{j}\left(S^{n-1} \cap S^{n-1}(b, \rho)\right)$ is a homeomorph of $S^{n-2}$ in $\partial D_{j}^{\prime}$ and by continuity $q(\Sigma) \leq q\left(S_{\rho}\right)<r$. We conclude that

$$
a(\Sigma) \leq \alpha\left(r ; \partial D_{j}\right)<\min \left\{\epsilon, d^{\prime}\right\} .
$$

We may assume the labeling is chosen so that

$$
C_{1}(\Sigma)=f_{j}\left(S^{n-1} \cap B^{n}(b, \rho)\right), \quad C_{2}(\Sigma)=f_{j}\left(S^{n-1} \backslash \bar{B}^{n}(b, \rho)\right) .
$$

But then $f_{j}(E) \subset C_{2}(\Sigma)$ and, by (46), $q\left(C_{2}(\Sigma)\right) \geq q\left(f_{j}(E)\right) \geq d^{\prime}$. It follows that $a(\Sigma)=q\left(C_{1}(\Sigma)\right)$. In particular, $q\left(f_{j}(x), f_{j}(b)\right) \leq a(\Sigma)<\epsilon$. Since this holds for each $x \in S^{n-1} \cap B^{n}\left(b, t^{2}\right)$ and for each $j \geq 1$ we conclude that $\left.\mathcal{F}\right|_{s^{n-1}}$ is equicontinuous at $b$ as desired. The proof is complete.

The converse of Lemma 7 is also true.

Lemma 8. For $j=1,2, \ldots$ let $f_{j}$ be a homeomorphism of $B^{n}$ into $\bar{R}^{n}$ mapping $B^{n} K$-quasiconformally onto a domain $D_{j}^{\prime}$. Assume that $\left\{f_{j}\right\}_{j=1}^{\infty}$ converges uniformly on compact sets of $D$ to a $K$-quasiconformal mapping $f$. If $\left\{f_{j}\right\}_{j=1}^{\infty}$ converges uniformly on $\bar{B}^{n}$ then

$$
\lim _{r \rightarrow 0^{+}} \sup _{j \geq 1} a\left(r ; \partial D_{j}^{\prime}\right)=0
$$

Proof. By Lemma 2 we know that $\lim _{r \rightarrow 0^{+}} \alpha\left(r ; D_{j}^{\prime}\right)=0$ for fixed $j \geq 1$. If (41) is not satisfied we may assume-passing to a subsequence and relabelingthat there is a $d>0$ and a homeomorph of $S^{n-2}, \Sigma_{j}^{\prime}$, in $\partial D_{j}^{\prime}$ so that $q\left(\Sigma_{j}^{\prime}\right) \rightarrow 0$, while

$$
a\left(\Sigma_{j}^{\prime}\right) \geq d
$$

for each $j \geq 1$. 

so that

Since $\mathcal{F}=\left\{f_{j}\right\}_{j=1}^{\infty}$ is uniformly equicontinuous on $\bar{B}^{n}$ there is a $t \in(0,1)$

$$
q\left(f_{j}(x), f_{j}(y)\right)<d / 2
$$

for each $j \geq 1$, whenever $x$ and $y$ in $\bar{B}^{n}$ satisfy $|x-y| \leq t$. Write $\Sigma_{j}=$ $f_{j}^{-1}\left(\Sigma_{j}^{\prime}\right)$. It follows from (51) and (52) that

$$
q\left(\Sigma_{j}\right) \geq t / 2
$$

for each $j \geq 1$. Now fix a continuun $F$ in $B^{n}$. Again using a result of Gehring [6]

$$
M\left(\Delta\left(F, \Sigma_{j}: B^{n}\right)\right) \geq 1 / 2 M\left(\Delta\left(F: \Sigma_{j}: \bar{R}^{n}\right)\right) \geq 1 / 2 \delta\left(s ; \bar{R}^{n}\right)=\delta_{0}>0,
$$

where $s=\min \{t / 2, q(F)\}$.

As the uniform limit of $\left\{f_{j}\right\}_{j=1}^{\infty}$ on $B^{n}, f$ is uniformly continuous on $B^{n}$, hence has an extension to a continuous map on $\bar{B}^{n}$, again denoted by 1 . From Lemma 5 we obtain (with $D_{0}^{\prime}=f\left(B^{n}\right)$ ):

$$
0<q\left(f(F), \partial D_{0}^{\prime}\right) \leq q\left(f(F), f\left(\Sigma_{j}\right)\right) \leq q\left(f\left(\Sigma_{j}\right)\right) \theta_{n, K}\left(\delta_{0}\right) .
$$

Thus

$$
q\left(f\left(\Sigma_{j}\right)\right)>d^{\prime}=1 / 2 q\left(/(F), \partial D_{0}^{\prime}\right) / \theta_{n, K^{(}}\left(\delta_{0}\right)
$$

for each $j \geq 1$.

However, since $q\left(\Sigma_{j}^{\prime}\right) \rightarrow 0$ and since $f_{j} \rightarrow f$ uniformly on $\bar{B}^{n}$ we can fix an index $j$ such that $q\left(\sum_{j}^{\prime}\right)<d^{\prime} / 3$ and such that $q\left(f_{j}(x), f(x)\right)<d^{\prime} / 3$ for all $x \in \bar{B}^{n}$. For $x$ and $y$ in $\Sigma_{j}$ we then obtain

$$
q(f(x), f(y)) \leq q\left(f(x), f_{j}(x)\right)+q\left(f_{j}(x), f_{j}(y)\right)+q\left(f_{j}(y), f(y)\right) \leq d^{\prime},
$$

whence $q\left(f\left(\Sigma_{j}\right)\right) \leq d^{\prime}$, contradicting (54). The contradiction shows that (41) must hold, as asserted. This completes the proof of Lemma 8.

We are now able to state:

Theorem 5. For $j=1,2, \ldots$, let $f_{j}$ be a bomeomorphism of $\bar{B}^{n}$ into $\bar{R}^{n}$ mapping $B^{n} K$-quasiconformally onto a domain $D_{j}^{\prime}$. Assume that $\left\{f_{j}\right\}_{j=1}^{\infty}$ converges uniformly on compact subsets of $B^{n}$ to K-quasiconformal map. The following are equivalent:

(i) $\left\{f_{j}\right\}_{j=1}^{\infty}$ converges uniformly on $\bar{B}^{n}$;

(ii) $\inf _{j \geq 1} \delta\left(r ; D_{j}^{\prime}\right)>0$, for each $r \in(0,1]$;

(iii) $\lim _{r \rightarrow 0^{+}} \sup _{j \geq 1} a\left(r ; \partial D_{j}^{\prime}\right)=0$. 
We recall that for nonempty, homeomorphic subsets $A$ and $B$ of $\bar{R}^{n}$, the Fréchet distance between $A$ and $B$, written $q_{F}(A, B)$, is defined by

$$
q_{F}(A, B)=\inf _{\theta} \sup _{x \in A} q(\theta(x), x),
$$

where the infimum is taken over the set of all homeomorphisms $\theta$ of $A$ onto $B$.

In general, it need not be the case that the limit map in Theorem 5 will map $B^{n}$ onto a Jordan domain. In the case that it does we obtain the following corollary to Theorem 5 and to Corollary 1 of [13]:

Corollary 3. For $j=1,2, \ldots$, let $f_{j}$ be a bomeomorphism of $\bar{B}^{n}$ into $\bar{R}^{n}$ mapping $B^{n} K$-quasiconformally onto a domain $D_{j}^{\prime}$. Suppose $\left\{f_{j}\right\}_{j=1}^{\infty}$ converges uniformly on compact subsets of $B^{n}$ to f, a K-quasiconformal map of $B^{n}$ onto a Jordan domain $D^{\prime}$. The following are equivalent:

(i) $\left\{f_{j}\right\}_{j=1}^{\infty}$ converges uniformly on $\bar{B}^{n}$;

(ii) inf $_{j \geq 1} \delta\left(r ; D_{j}^{\prime}\right)>0$, for each $r \in(0,1]$;

(iii) $\lim _{r \rightarrow 0^{+}} \sup _{j \geq 1} \alpha\left(r ; \partial D_{j}^{\prime}\right)=0$;

(iv) $\lim _{j \rightarrow \infty}^{r \rightarrow 0} q_{F}\left(\partial D^{\prime}, \partial D_{j}^{\prime}\right)=0$.

We remark that the implications (i) $\Leftrightarrow$ (iii) in Corollary 3 give an $n$ dimensional analogue to a result of Gaier [3], which, in turn, is based on a clas. sical theorem of Courant [1]. The implications (i) $\Leftrightarrow$ (iv) represent an $n$ dimensional version of a theorem of Radó [14].

\section{REFERENCES}

1. R. Courant, Über eine Eigenschaft der Abbildungsfunktionen bei konformer Abbildung, Göttinger Nachr. 1922, 69-70.

2. O. J. Farrell, On approximation to a mapping function by polynomials, Amer. J. Math. 54 (1932), 571-578.

3. D. Gaier, Über die konforme Abbildung veränderlicher Gebiete, Math. Z. 64(1956), 385-424. MR 17, 1191 .

4. F. W. Gehring, Symmetrization of rings in space, Trans. Amer. Math. Soc. 101 (1961), 499-519. MR 24 \#A2677.

5. - The Carathéodory convergence theorem for quasiconformal mappings in space, Ann. Acad. Sci. Fenn. Ser. A I No. 336 (1963), 1-21. MR 28 \#4107.

6. - Lectures on quasiconformal mappings, Mittag-Leffler Institute, 1972 (unpublished).

7. F.W. Gehring and J. Väisälä, The coefficien ts of quasiconformality of domains in space, Acta Math. 114 (1965), 1-70. MR 31 \#4905.

8. O. Lehto and K. I. Virtanen, Quasikonforme Abbildungen, Die Grundlehren der math. Wissenschaften in Einzeldarstellungen mit besonderer Berücksichtigung der Anwendungsgebiete, Band 126, Springer-Verlag, Berlin and New York, 1965. MR 32 \#5872.

9. R. Näkki, Continuous boundary extension of quasiconformal mappings, Ann. Acad. Sci. Fenn. Ser. A I No. 511 (1972), 2-9. 
10. R. Näkki, Extension of Loewner's capacity theorem, Trans. Amer. Math. Soc. (to a ppear).

11. R. Näkki and B. Palka, Uniform equicontinuity of quasiconformal mappings, Proc. Amer. Math. Soc. 37 (1973), 427-433.

12. B. Palka, Boundary distortion and the uniform convergence of quasiconformal mappings, Thesis, University of Michigan, Ann Arbor, Mich., 1972.

13. - Fréchet distance and the uniform convergence of quasiconformal mappings, Duke Math. J. 39 (1972), 289-296.

14. T. Radó, Sur la représentation conforme des domaines variables, Acta Sci. Math. Szeged 1 (1922/23), 180-186.

15. J. Väisälä, Lectures on n-dimensional quasiconformal mappings, Lecture Notes in Math, vol. 229, Springer-Verlag, Berlin and New York, 1970.

DEPARTMENT OF MATHEMATICS, BROWN UNIVERSITY, PROVIDENCE, RHODE ISLAND 02912 